\title{
Use of PET tracers for parathyroid localization: a systematic review and meta-analysis
}

\author{
Wouter P. Kluijfhout ${ }^{1,2}$. Jesse D. Pasternak ${ }^{3}$ - Frederick Thurston Drake ${ }^{1}$. \\ Toni Beninato ${ }^{1}$ - Jessica E. Gosnell ${ }^{1}$ - Wen T. Shen ${ }^{1}$ • Quan-Yang Duh ${ }^{1}$ • Isabel E. Allen ${ }^{4}$. \\ Menno R. Vriens ${ }^{2} \cdot$ Bart de Keizer $^{5} \cdot$ Miguel H. Pampaloni $^{6} \cdot \operatorname{Insoo}$ Suh $^{1}$
}

Received: 12 February 2016 / Accepted: 3 April 2016/Published online: 16 April 2016

(C) The Author(s) 2016. This article is published with open access at Springerlink.com

\begin{abstract}
Purpose The great spatial and temporal resolution of positron emission tomography might provide the answer for patients with primary hyperparathyroidism (pHPT) and non-localized parathyroid glands. We performed a systematic review of the evidence regarding all investigated tracers.

Methods A study was considered eligible when the following criteria were met: (1) adults $\geq 17$ years old with non-familial pHPT, (2) evaluation of at least one PET isotope, and (3) postsurgical and pathological diagnosis as the gold standard. Performance was expressed in sensitivity and PPV.

Results Twenty-four papers were included subdivided by radiopharmaceutical: 14 studies investigated $\mathrm{L}-\left[{ }^{11} \mathrm{C}\right]$ Methionine (11C-MET), one $\left[{ }^{11} \mathrm{C}\right] 2$-hydroxy- $N, N, N$-trimethylethanamium (11C-CH), six 2-deoxy-2- $\left[{ }^{18} \mathrm{~F}\right]$ fluoro-D-glucose (18F-FDG), one $6-\left[{ }^{18} \mathrm{~F}\right]$ fluoro-L-DOPA (18F-DOPA), and three $N$-[( $\left.{ }^{18} \mathrm{~F}\right)$ Fluoromethyl]-2-hydroxy- $N, N$-dimethylethanaminium (18F-FCH). The 14 studies investigating MET included a total
\end{abstract}

Wouter P. Kluijfhout

wpkluijfhout@gmail.com

1 Department of Surgery, University of California San Francisco, San Francisco, CA, USA

2 Department of Surgery, University Medical Center Utrecht, Utrecht, Netherlands

3 Department of Surgery, University Health Network, Toronto, ON, Canada

4 Department of Epidemiology and Biostatistics, University of California San Francisco, San Francisco, CA, USA

5 Department of Radiology and Nuclear Medicine, University Medical Center Utrecht, Utrecht, Netherlands

6 Department of Nuclear Medicine, University of California San Francisco, San Francisco, CA, USA of 327 patients with 364 lesions. Sensitivity for the detection of a lesion in the correct quadrant had a pooled estimate of $69 \%$ (95\% CI 60-78\%). Heterogeneity was overall high with $\mathrm{I}^{2}$ of $51 \%(p=0.01)$ for all 14 studies. Pooled PPV ranged from 91 to $100 \%$ with a pooled estimate of $98 \%$ (95\% CI 96-100\%). Of the other investigated tracers, 18 -FCH seems the most promising with high diagnostic performance.

Conclusions The results of our meta-analysis show that 11CMET PET has an overall good sensitivity and PPV and may be considered a reliable second-line imaging modality to enable minimally invasive parathyroidectomy. Our literature review suggests that $18 \mathrm{~F}-\mathrm{FCH}$ PET may produce even greater accuracy and should be further investigated using both low-dose $\mathrm{CT}$ and MRI for anatomical correlation.

Keywords Primary hyperparathyroidism $\cdot$ Minimal invasive parathyroidectomy $\cdot$ PET/CT $\cdot 11 \mathrm{C}$-Methionine .

18F-Fluorocholine

\section{Introduction}

Primary hyperparathyroidism (pHPT) is the third most common endocrine disorder with a prevalence of around 4 per 1000. Incidence rises with age and is twice as common in women. Patients can present with a wide variety of welldescribed symptoms, ranging from nausea and fatigue to severe osteoporosis and cardiovascular complications [1]. Diagnosis is established biochemically, with the finding of relatively elevated serum calcium $(\mathrm{Ca})$ level and concomitant inappropriately elevated parathyroid hormone (PTH) level. Most commonly, only one of the four glands is abnormaltypically an adenoma - and causes the condition (75-85\%). Less frequently, there are multiple adenomas (15-25\%) and very rarely a carcinoma (1\%) [2]. There is a universal 
agreement that all patients with symptoms, significant signs of disease (renal or bone manifestations), or young age ( $<50$ years) should undergo surgical exploration [3]. Over the past decade, there has been a shift towards minimally invasive parathyroidectomy (MIP); a focused operation whereby only one parathyroid is removed. Compared to the conventional neck exploration, in which all four glands are investigated intra-operatively, MIP is associated with shorter operating time, lower complication rates, and smaller incision size $[4,5]$.

Imaging is critical in order to enable successful MIP. Aside from its ability to localize the pathological gland(s), accurate imaging provides valuable anatomical information for the surgeon. ${ }^{99} \mathrm{Tc}$-sestamibi single photon emission computed tomography (SPECT-CT) has become one of the mainstays in first-line parathyroid imaging based on robust evidence demonstrating its ability to predict the location of an adenoma [6]. However, a recent meta-analysis showed that its sensitivity is only $63-84 \%$ (depending on protocol), which leaves a substantial amount of cases with equivocal imaging results [7]. For these cases, several second-line imaging modalities like MRI and CT are used, which have variable performance characteristics. Another modality that has been studied is positron emission tomography (PET). The greater spatial and temporal resolution of PET compared to SPECT imaging allows detection of even the smallest pathological glands, which in theory could improve sensitivity. One of the first reported uses of PET for parathyroid disease used 2-deoxy-2- $\left[{ }^{18} \mathrm{~F}\right]$ fluoro-Dglucose (18F-FDG), which revealed the location of a pathological parathyroid gland in a patient with $\mathrm{pHPT}$ [8]. Over the years, several other tracers have been investigated as well, with variable results. The performance of PET depends mostly on the ability of the tracer to show specific uptake in the targeted organ. Although PET seems to be a promising new possibility for imaging of pathologic parathyroid glands, not all radiopharmaceuticals are suitable based on multiple factors, including tracer half-life, specificity to parathyroid uptake, and individual hospital characteristics. To our knowledge, there is no pre-existing literature that has reviewed all of the investigated PET tracers in patients with pHPT. Therefore, we performed a systematic review of the evidence available regarding all investigated tracers and also performed a meta-analysis of the data regarding the use of $\mathrm{L}-\left({ }^{11} \mathrm{C}\right)$ Methionine (11C-MET), the most extensively investigated tracer thus far.

\section{Material and methods}

\section{Literature search}

We performed a search of the Embase, PubMed, and Cochrane Library databases published through March 18,
2016, to identify studies investigating the diagnostic value of all PET radiopharmaceuticals for parathyroid localization in patients with biochemical pHPT. We employed an extensive search filter by using all relevant synonyms and Mesh/Emtree terms (Table 1). We completed our search by performing a robust cross-reference check in Web of Science and manually searched references of selected articles, related reviews, and guidelines.

\section{Inclusion and exclusion criteria}

From the publications retrieved via our systematic search, we removed any duplicates (Fig. 1). All remaining unique publications were screened based on title and abstract for eligibility. A study was considered eligible when the following criteria were met: (1) adults $\geq 17$ years old with biochemical non-familial pHPT, (2) evaluation of at least one PET tracer, and (3) post-surgical and pathological diagnosis as the gold standard. If an article presented data for multiple study groups, of which some were eligible for inclusion, the eligible study groups were included if their pertinent data could be extracted.

We excluded studies that differed in design (case reports, systematic reviews), domain (other than $\mathrm{pHPT}$ ), and language (non-English). All titles and abstracts were screened

Table 1 Search strategy

\begin{tabular}{|c|c|}
\hline $\begin{array}{l}\text { Strategy component } \\
\text { and step no. }\end{array}$ & Query \\
\hline \multicolumn{2}{|c|}{ PubMed and Cochrane database } \\
\hline \multicolumn{2}{|l|}{ Patient } \\
\hline 1 & $\begin{array}{l}\text { Hyperparathyroidism OR parathyroid OR HPT } \\
\text { OR PHPT }\end{array}$ \\
\hline 2 & $\begin{array}{l}\text { MeSH descriptor hyperparathyroidism explode } \\
\text { all trees }\end{array}$ \\
\hline \multicolumn{2}{|l|}{ Intervention } \\
\hline 3 & PET OR "Positron Emission Tomography" \\
\hline 4 & $\begin{array}{l}\text { MeSH descriptor Tomography, Emission- } \\
\text { Computed explode all trees }\end{array}$ \\
\hline \multicolumn{2}{|l|}{ Embase } \\
\hline \multicolumn{2}{|l|}{ Patient } \\
\hline 1 & $\begin{array}{l}\text { Hyperparathyroidism OR parathyroid OR HPT } \\
\text { OR PHPT }\end{array}$ \\
\hline 2 & EMTREE hyperparathyroidism explosion \\
\hline \multicolumn{2}{|l|}{ Intervention } \\
\hline 3 & PET OR "Positron Emission Tomography" \\
\hline 4 & $\begin{array}{l}\text { EMTREE positron emission tomography } \\
\text { explosion }\end{array}$ \\
\hline \multicolumn{2}{|l|}{ Merge } \\
\hline 5 & $1 \mathrm{OR} 2$ \\
\hline 6 & 3 OR 4 \\
\hline 7 & 5 AND 6 \\
\hline
\end{tabular}


Fig. 1 Flowchart of study inclusion

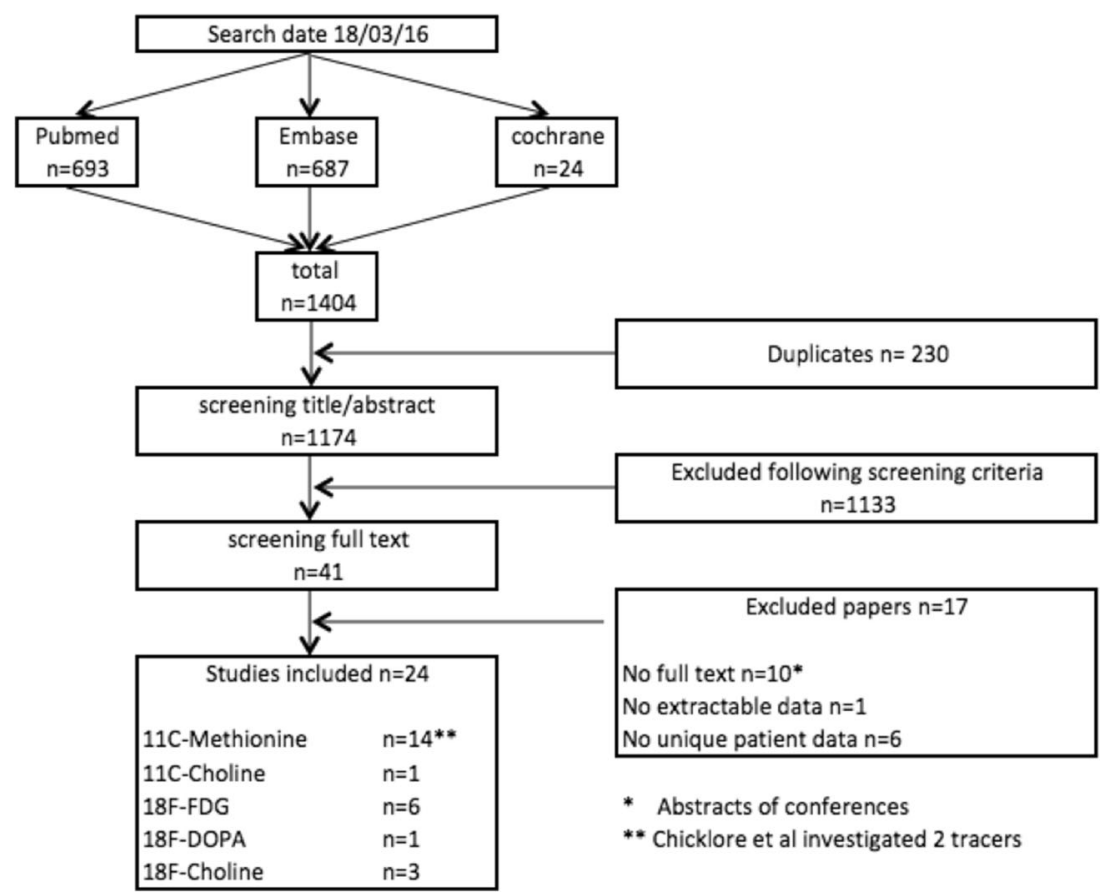

independently by two authors (W.P.K. and J.D.P.). Any disagreements in selection were discussed, and after consensus, the selected papers were screened for full text review.

Studies were included in the systematic review if they investigated at least five patients, independent of the tracer. Studies were included in the meta-analysis if the following inclusion criteria were met: (1) use of the tracer 11C-MET, (2) extractable and unique data for the calculation of sensitivity and PPV for localization of pathological gland(s) to the correct quadrant (left lower, right lower, left upper, right upper, or ectopic), (3) histological examination of the resected parathyroid gland(s), and (4) a minimum of five included patients.

\section{Quality assessment}

To assess the quality of the included studies, we used the Quality Assessment of Studies of Diagnostic Accuracy Included in Systematic Reviews (QUADAS-)2 tool [9]. This tool is designed to assess the quality of primary diagnostic accuracy studies. It consists of 4 key domains that address (1) patient selection, (2) index test, (3) reference standard and (4) flow of patients through the study and timing of the index tests and reference standard. The domains are assessed for risk of bias and applicability. The two reviewers (W.P.K. and J.P.) used this tool to independently evaluate the methodological quality of the included studies. Disagreements were solved by discussion and thus the final report is based upon consensus of the two reviewers.

\section{Data extraction}

Data were extracted using a standardized spreadsheet. Study design characteristics included the tracer used, injected dose, possible combination with CT, study population, and number of included patients. Since pHPT is a biochemical diagnosis and all patients included were diagnosed with pHPT, there were no true negative scans and therefore specificity and NPV were not calculated. The performance of PET was expressed in sensitivity (total number of glands localized to the correct quadrant/total number of pathological glands found during surgery) and PPV (total number of glands localized to the correct quadrant/ total number of glands suspicious for adenomas or hyperplasia). Data regarding MET was calculated using pooled proportion and displayed using forest plots. Sensitivity was defined as the probability that the localization study correctly identified the pathological gland(s). PPV was defined as the probability that a patient with a positive localization study had a pathological gland in that specific location. If data regarding sensitivity or PPV was missing in the selected paper, the author was contacted. Since sensitivity and PPV were calculated using the aforementioned criteria for patients with $\mathrm{pHPT}$, the results may differ from the original papers.

For the studies investigating MET, random-effects models (REM) were used to pool the data [10]. The REM model was fit to account for study heterogeneity. The heterogeneity among studies was tested using the $\mathrm{I} 2$ statistic test with a $p$ value of less than 0.1 for statistical significance [11]. The I2 statistic is expressed in a percentage scale in which $0 \%$ 
implies study homogeneity and $100 \%$ indicates that betweenstudy variance is much larger than within study variance. The investigated study population could have influenced the diagnostic performance, thereby causing heterogeneity, and was therefore investigated by subgroup-analysis. The studies were subdivided into 3 groups consisting of patients (1) without specific selection, (2) with negative or discordant conventional imaging, and (3) with a history of previous parathyroid surgery. Funnel plots were used as a visual tool for investigating heterogeneity and publication bias [12]. These plots are scatterplots of the treatment effects estimated from individual studies against a measure of study size. In the absence of publication bias, the funnel plots are symmetrical.

Data analysis was performed using StataSE 13 and conducted by a biostatistician specializing in meta-analysis (I.A.). For this type of study, no formal consent is required.

\section{Results}

\section{Literature search}

The literature search yielded a total of 1174 unique papers, of which 41 papers remained eligible after screening of title and abstract for relevance. After full text review, 24 papers were included. These were subdivided by radiopharmaceutical: 14 studies investigated $11 \mathrm{C}-\mathrm{MET}$, one $\left[{ }^{11} \mathrm{C}\right] 2$-hydroxy- $N, N, N$ trimethylethanamium $(11 \mathrm{C}-\mathrm{CH})$, six $18 \mathrm{~F}-\mathrm{FDG}$, one $6-\left[{ }^{18} \mathrm{~F}\right]$ fluoro-L-DOPA (18F-DOPA), and three $N$-[ $\left({ }^{18} \mathrm{~F}\right)$ Fluoromethyl]2-hydroxy- $N, N$-dimethylethanaminium (18F-FCH) [13-36]. Excluded were studies that had fewer than five patients, abstracts from conferences without available full text, and redundant studies investigating the same population. The process of study selection is illustrated in Fig. 1. The 14 studies investigating MET underwent meta-analysis, which included a total of 327 patients with 364 lesions.

\section{Characteristics of the MET studies}

All studies were single-center and included between eight and 29 patients, with the exception of the study by Weber et al., which included 102 patients. Four studies had a prospective design, whereas the rest were conducted retrospectively. Most studies also involved patients with secondary (sHPT) or tertiary HPT, or patients that did not proceed to surgery (our gold standard). These patients were excluded from the analysis. Patients with negative or discordant conventional imaging were included in three studies, and two other studies specifically included patients with previous neck surgery. The injected dose of radioactive tracer varied between 370 and $1100 \mathrm{MBq}(10-30 \mathrm{mCi})$ and scanning started between 0 and 40 min after injection with an additional low-dose CT in ten studies. The characteristics are listed in Table 2.

\section{Quality assessment of the MET studies}

Quality assessment is displayed in Fig. 2 using the QUADAS 2 tool for risk of bias and applicability. Overall, there was a particularly high risk of bias arising from lack of a reference standard and heterogeneity in patient selection. Bias related to the reference standard relates to the fact that a clear definition of cure was lacking in several of the studies. The reference standard varied between no mention of follow-up data to documentation of normocalcemia at 6 months, which is the widely excepted definition of cure. Since estimates of accuracy are based on the assumption that the reference standard is $100 \%$ sensitive and specific, the lack of a clear reference standard might have resulted in misinterpretation of the accuracy of the index test. Substantial risk of bias was present since most studies did not enroll consecutive patients or a random sample of eligible patients. Moreover, as can be seen in Table 2, only a proportion of patients included in the studies underwent surgery. (Because of this, some studies were excluded from the meta-analysis, since they only performed surgery on patients with a positive scan, potentially overestimating its performance.) Concerns regarding applicability were low for the index test, as the scans were usually conducted according to a specific protocol within the studies. However, there was great variability between studies with regard to the PET protocol in terms of tracer dose, use of additional CT, and timing of the scan, which raises concerns as to the applicability of the index test. Patient selection also raises applicability concerns, since the study populations of patients were variable and could not entirely be adjusted for via sub-analysis.

Publication bias was investigated using a funnel plot. The distribution indicates a publication bias of small and most likely irrelevant studies (Fig. 3).

\section{Accuracy and heterogeneity of the MET studies}

Individual and summary estimates of per-quadrant sensitivity and PPV are shown in Fig. 4a and b. Sensitivity for the detection of a lesion in the correct quadrant ranged from 44 to $91 \%$, with a pooled estimate of $77 \%$ (95\% CI 71-84\%). Subgroup-analysis based on selection of included patients was performed as well. A total of 9 studies did not specifically select patients (group 1) and showed a random pooled sensitivity of $78 \%$ (95\% CI 70-86\%), which was almost the same as for the three studies that included patients with negative or inconclusive conventional imaging (group 2) and had a pooled sensitivity of $81 \%$ (95\% CI 70-91\%). The two studies that predominantly investigated patients with previous parathyroid surgery had discrepant outcomes with sensitivities of 54 and $84 \%$. Heterogeneity was overall high with $\mathrm{I}^{2}$ of $51 \%(p=0.01)$ for all 14 studies and moderate between the 2 subgroups $\left(\mathrm{I}^{2}=42 \% ; p=0.06\right)$ (Fig. $4 \mathrm{a}$ and b). Pooled PPV 
Table 2 Characteristics of included studies investigating 11C-Methionine

\begin{tabular}{lcccllll}
\hline \multirow{2}{*}{$\begin{array}{l}\text { Study } \\
\text { First author }\end{array}$} & Year & \multicolumn{2}{l}{ No. of patients } & Study population & Dose (MBq) & CT & Time (min) \\
\cline { 2 - 6 } & & Total & pHPT + surgery & & & \\
\hline Braeuning & 2015 & 18 & 12 & Neg/disc imaging & 600 & yes & 20 \\
Hayakawa & 2015 & 23 & 15 & Mixed & $441-906$ & yes & $15-34$ \\
Chicklore & 2014 & 43 & 15 & Mixed & 740 & yes & 10 \\
Martinez & 2014 & 14 & 14 & Mixed & 740 & yes & 10 and 40 \\
Weber & 2013 & 102 & 102 & Mixed & $?$ & yes & 20 \\
Chun & 2013 & 16 & 8 & Neg/disc imaging & $370-444$ & yes & $30-40$ \\
Schalin & 2012 & 21 & 21 & Previous surgery & 440 & yes & 20 \\
Oksuz & 2011 & 8 & 8 & Mixed & 700 & $4 / 4$ & 15 \\
Weber & 2010 & 33 & 33 & Mixed & $?$ & yes & 20 \\
Herrmann & 2009 & 41 & 9 & Mixed & 430 & no & $15-20$ \\
Tang & 2008 & 30 & 22 & Mixed & 555 & yes & 20 \\
Beggs & 2005 & 51 & 29 & Neg/disc imaging & 619 & no & 15 \\
Otto & 2004 & 30 & 14 & Mixed & $900-1100$ & no & 10 \\
Sundin & 1996 & 34 & 25 & Previous surgery & 750 & no & direct \\
\hline
\end{tabular}

pHPT primary hyperparathyroidism, Neg negative, Disc discordant

${ }^{a}$ Previous surgery in 25/34 patients included in the study ranged from 91 to $100 \%$ with a pooled estimate of $98 \%$ (95\% CI 96-100\%) (Fig. 5).

\section{Other investigated PET tracers}

Details about studies investigating other PET tracers are listed in Table 3. From six studies investigating FDG, three were from the same author; however, the domain differed. Two of these included only patients without previous parathyroidectomy - consisting of 17 and 21 patients with $\mathrm{pHPT}$ - and excluded patients with hyperplasia from the results $[33,36]$. These studies found sensitivities of 94 and $86 \%$ and PPVs of 85 and $86 \%$, respectively. The third included 20 patients with a history of neck surgery and found a much lower sensitivity and PPV, both $62 \%$ [32]. The three other studies found even lower sensitivities, varying between 0 and $27 \%$, although PPVs were consistently high. Notably, none of the 8 scans performed by Sisson et al. showed a focus that could be attributed to a parathyroid adenoma [35].

Five recent studies published results regarding $18 \mathrm{~F}-\mathrm{FCH}$; however, Michaud et al. and Lezaic et al. published two articles using the same population $[28,29,37,38]$. The most recent study of Michaud et al. included 17 patients without prior neck surgery, divided in 11 patients with $\mathrm{pHPT}$, five with sHPT, and one lithium-associated [28]. All patients had 18FFCH PET-CT because of negative/discordant ultrasound and ${ }^{123} \mathrm{I}{ }^{99 \mathrm{~m}} \mathrm{Tc}$-sestamibi subtraction scintigraphy. Sensitivity per lesion was up to $94 \%$. The most recent study of Lezaic et al. included 43 patients with $\mathrm{pHPT}$ and no previous neck surgery [29]. All patients underwent ultrasound, ${ }^{99 \mathrm{~m}} \mathrm{Tc}$-sestamibi/ pertechnetaat subtraction scintigraphy, SPECT-CT, and 18FFCH PET-CT. Sensitivity per lesion of PET-CT was up to $95 \%$. Kluijfhout et al. conducted 5 scans in patients with negative conventional imaging and found a sensitivity of $80 \%$ with a PPV of $100 \%$ [27].
Fig. 2 QUADAS 2 tool for risk of bias and applicability

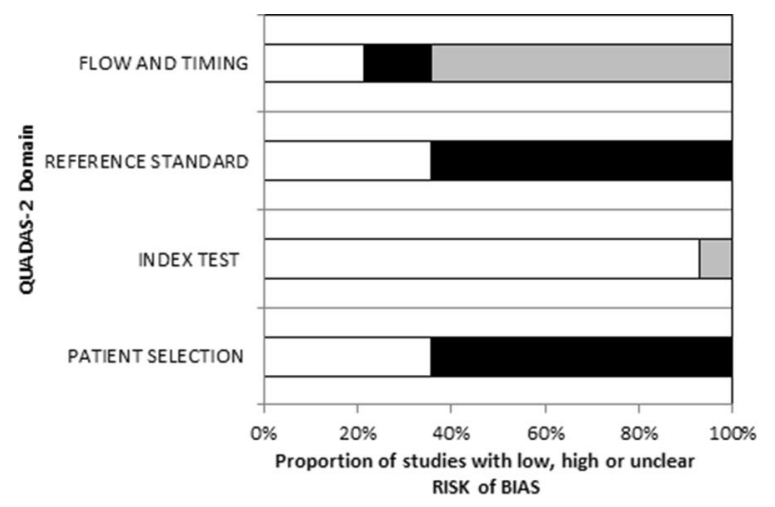

口Low $\mathbf{m}$ High $\square$ Unclear

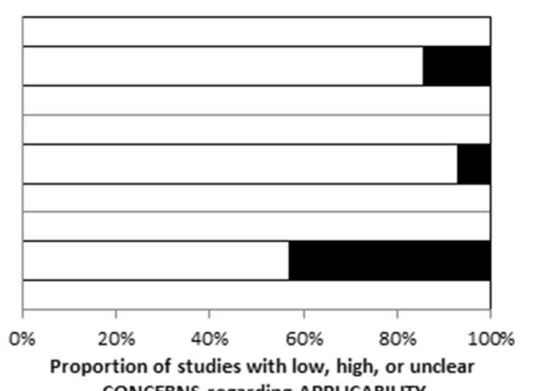

CONCERNS regarding APPLICABILITY 


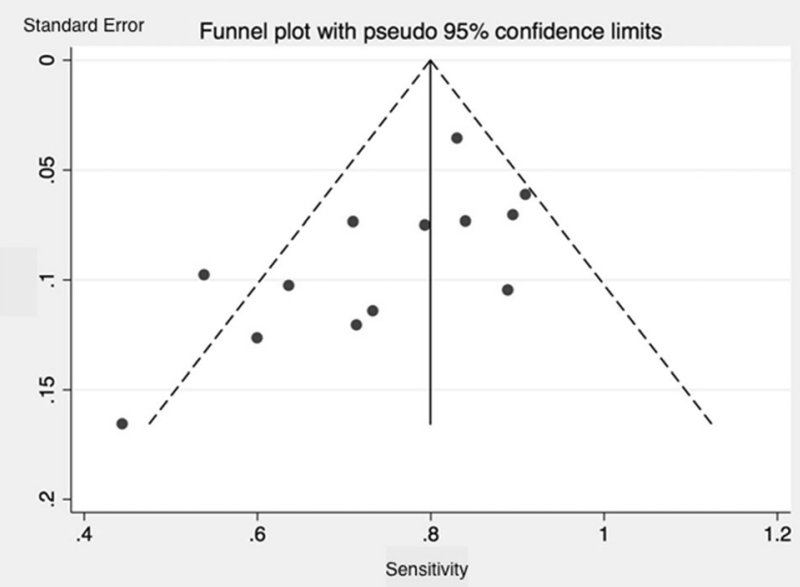

Fig. 3 Funnel plot of 11C-Methionine studies included in this metaanalysis. The distribution indicates a publication bias of small and most likely irrelevant studies

Lange-Nolde et al. investigated the performance of $18 \mathrm{~F}$ DOPA [31]. They included eight patients with pHPT. All had histologically proven adenomas; however, none of the scans showed any detectable uptake.

Orevi et al. published preliminary results of $11 \mathrm{C}-\mathrm{CH}$ PETCT [30]. They included 40 patients with HPT, of which 20 were diagnosed with pHPT and the latter predominantly with sHPT. In 24 of 27 patients that underwent surgery so far, there was concordance between PET result and surgical findings. There was insufficient data to calculate specific performance for patients with $\mathrm{pHPT}$; however, the scan was clearly positive in 37 out of 40 patients.

\section{Discussion}

We performed a meta-analysis to investigate the performance of 11C-MET PET to localize pathological parathyroid gland(s) in patients with pHPT. Our results show a pooled sensitivity and PPV for the detection of a pathological parathyroid in the correct quadrant of 77 and $98 \%$, respectively. In subgroup-analysis, we found no difference in sensitivity in patients without specific selection versus patients with negative/discordant conventional imaging (78 versus $81 \%$, respectively). Among the other investigated tracers in our systematic review, $18 \mathrm{~F}-\mathrm{FCH}$ seems the most promising with sensitivity ranging from $80-100 \%$ and PPV $89-100 \%$ in three studies with a total of 37 patients.

Our pooled sensitivity of $77 \%$ is slightly lower than the pooled sensitivity of $81 \%$ found by Caldarella et al. who published a meta-analysis regarding 11C-MET PET in 2012 [39]. Since then, new evidence has been published and there are several major methodological differences between that study and the current meta-analysis. First, Caldarella et al. did not solely include patients with pHPT but also with
SHPT. Second, their calculations are based on a per-patient level: as most surgeons favor MIP to bilateral neck exploration, a per-lesion sensitivity for localization to the correct quadrant may be more clinically relevant. Identification of the right quadrant per lesion is more precise, which might explain our slightly lower pooled sensitivity. Lastly, PPV is not calculated; instead, the authors presented a detection rate, defined as percentage of positive scans, without correlating this to the surgical outcome. In an attempt to examine a more homogenous group, we included only patients with pHPT. The domain of the included patients did however still vary greatly, which is why subgroup-analysis was performed to examine whether patient domain influenced the accuracy of 11C-MET PET.

In subgroup-analysis, performance of 11C-MET PET in a mixed patient cohort (group 1; pooled sensitivity $78 \%$ ) was comparable to conventional ${ }^{99 \mathrm{~m}} \mathrm{TC}$-sestamibi SPECT (sensitivity $79 \%$ ), but substantially lower compared to sestamibi SPECT/CT (sensitivity of $88 \%$ ) [40, 41]. Remarkably, the performance of 11C-MET PET did not decline significantly when only patients with negative or inconclusive imaging were included (group 2, pooled sensitivity $81 \%$ ). This suggests that 11C-MET PET is relatively most useful in a substantial amount of cases with negative/discordant conventional imaging and could therefore be considered as a suitable second-line imaging modality.

The ability of 11C-MET PET to detect hyperplastic glands was lower compared to patients with adenomas. In the largest study included in our meta-analysis, overall sensitivity was $83 \%$, versus $33 \%$ for hyperplastic glands [17]. Hyperplastic glands are often smaller in size and weigh less than adenomas, both factors that have previously been associated with decreased sensitivity [14]. This might also be a contributing reason as to why performance is lower in patients with persistent pHPT after previous parathyroid surgery. Schalin et al. included 21 patients with $\mathrm{pHPT}$ and persistent disease and found a sensitivity of $44 \%$, substantially lower than the pooled sensitivity of $77 \%$ found in this study [19]. Weber et al. also performed a subgroup-analysis on patients with previous neck surgery, including patients after thyroid surgery. Remarkably the sensitivity in this group was higher compared to the total group (94 versus $83 \%$ ). Thyroid tissue reduces the lesion-to-background ratio due to physiological thyroidal uptake of 11C-MET and thereby complicates reading of parathyroid scans [22]. Pathological parathyroids that are in a juxtathyroidal location can therefore cause false negative outcomes [26].

Detection of accumulated 11C-MET can be done at different times after injection, and protocols varied in this study from scanning immediately after injection to scanning 40 min post-injection. Two studies had protocols in which patients were scanned twice at 10 and $40 \mathrm{~min}$ to compare the effect of timing on diagnostic efficacy $[16,25]$. They found the best parathyroid to background contrast and the best 
Fig. 4 a Forest plot of sensitivity of all studies. The pooled result is displayed by the vertical line. Horizontal lines indicate the $95 \%$ confidence intervals of each separate study. Size of the square is directly linked to the number of patients included in the study. $\mathbf{b}$ Forest plot of sensitivity of subgroup-analysis depending on domain of included patients. The pooled result is displayed by the vertical line. Horizontal lines indicate the $95 \%$ confidence intervals of each separate study. Size of the square is directly linked to the number of patients included in the study. $S E$ sensitivity, $C I$ confidence interval

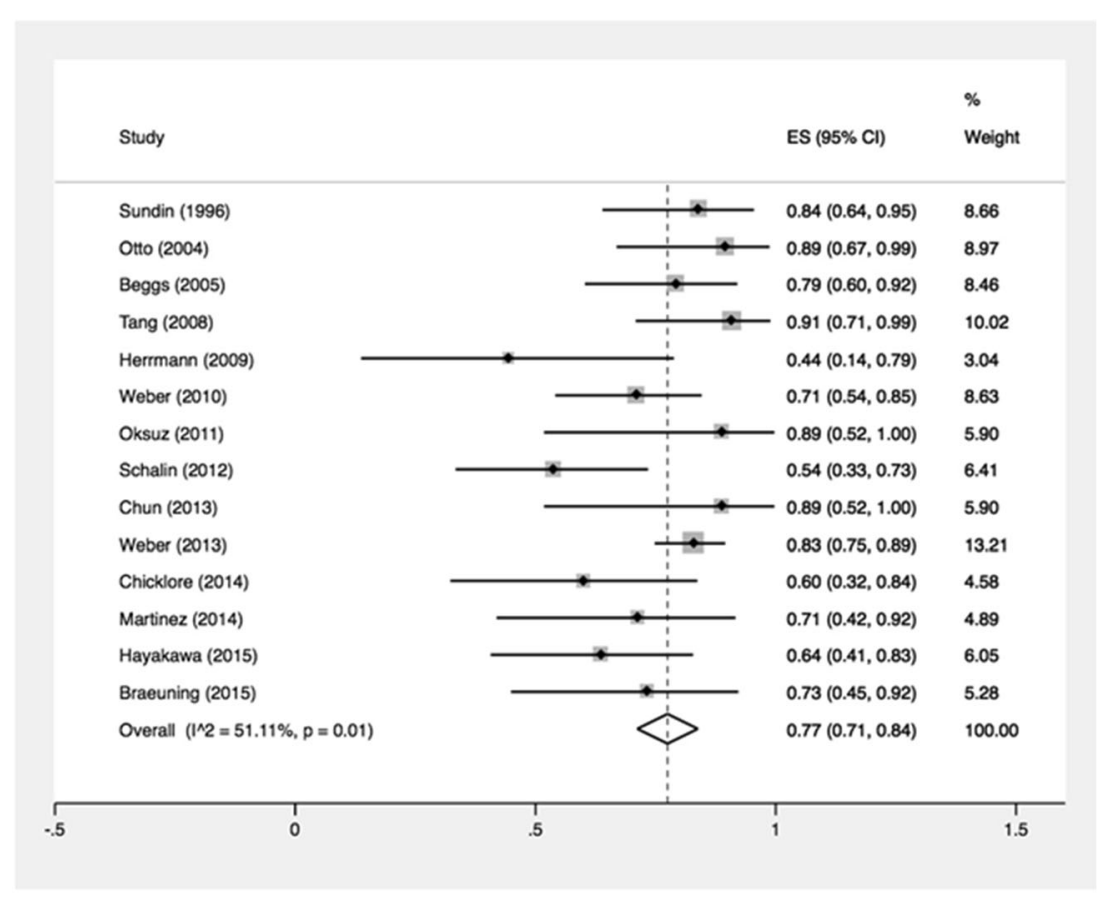

a

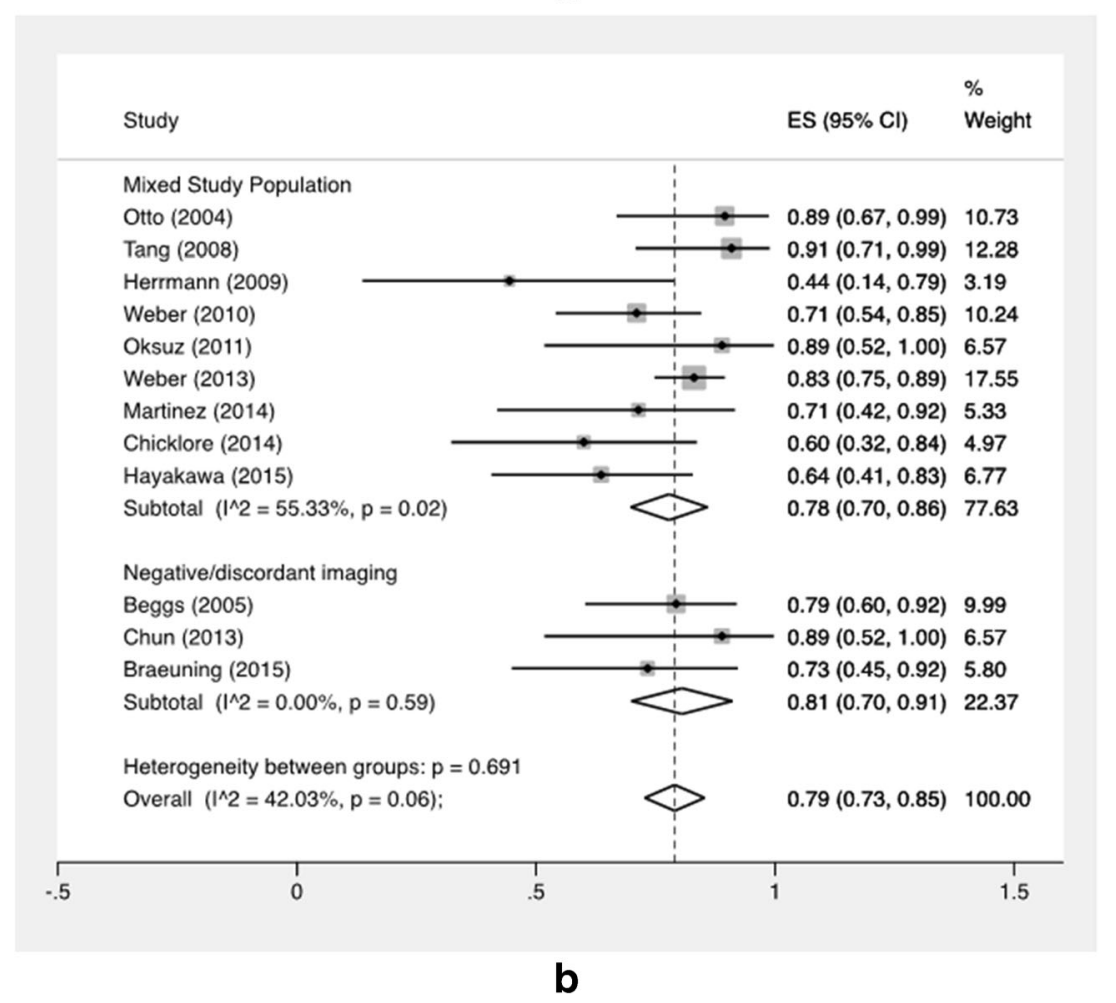

delineation of hyper-functioning parathyroid after $10 \mathrm{~min}$, although the best parathyroid to thyroid contrast was seen after $40 \mathrm{~min}$. Martinez et al. included the late scan when the 10-min scan was negative, which resulted in the detection of one additional positive parathyroid gland in their study. Scans later than 40 min resulted in insufficient count statistics owing to the short half-life of 11C-MET (20 min). The short half-life of
$11 \mathrm{C}-\mathrm{MET}$ and the complicating labeling procedure is also one of the biggest limitations of MET. It requires the center to have a cyclotron for production of the tracer since transportation from another facility would take too much time.

Recent publications often used a combination of PET and CT. Contrast enhancement CT can be useful to differentiate with lymph nodes and might therefore prevent false positive 
Fig. 5 Forest plot of positive predictive value. $S E$ sensitivity, $C I$ confidence interval

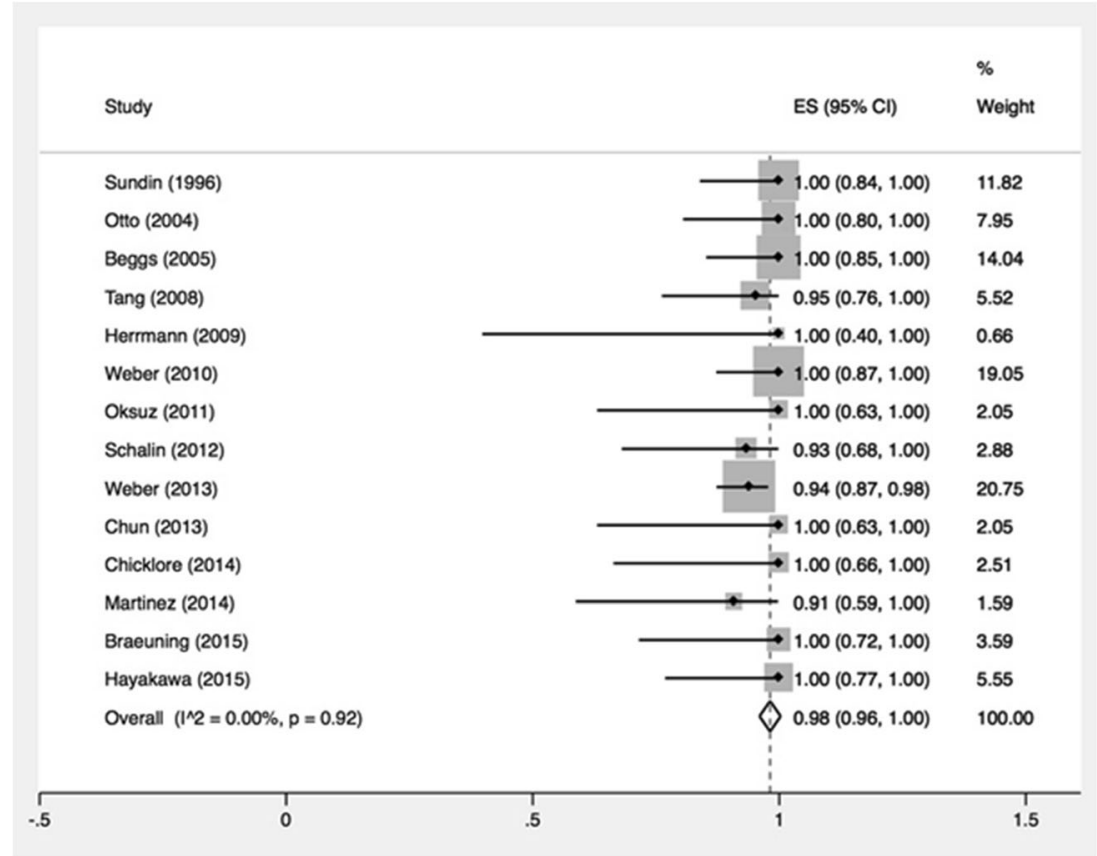

outcomes [42]. However, there is also increasing attention to the possible risks of extra radiation involved with CT [43]. Several studies also investigated other factors that may affect the accuracy, such as goiter, thyroiditis, and parathyroid hormone level; however, a separate analysis could not be performed due to insufficient available data. Nevertheless, in general PET appears to work well even in these settings [17, 26].

The second best investigated tracer is $18 \mathrm{~F}-\mathrm{FDG}$, which has the advantage of a significant longer half-time of $110 \mathrm{~min}$, enabling off-site production. Also, it is by far the most commonly used PET radiopharmaceutical and has wide availability. These benefits notwithstanding performance varied greatly between the six studies that have investigated its use with a sensitivity of 0-94 \% and PPV of 62-100\%. Due to these highly discrepant findings, FDG appears to be a less useful tracer for the detection of pathological parathyroids. 18F-DOPA, in a limited trial, performed even more poorly. Using a prospective study design, Lange-Nolde et al. scanned eight patients with pHPT, each of whom had adenomas detected in the operating room; however, none of the 18F-DOPA PET scans were positive [31]. More promising seems to be the use of $11 \mathrm{C}-\mathrm{CH}$. A recent prospective study by Orevi et al. included 40 patients with HPT, and there were 37 choline positive scans [30]. Although their results are preliminary and only some of the patients have undergone surgery, these

Table 3 Characteristics of studies investigating PET tracers other than 11C-Methionine

\begin{tabular}{|c|c|c|c|c|c|c|c|c|c|}
\hline \multirow{2}{*}{$\begin{array}{l}\text { Study } \\
\text { First author }\end{array}$} & \multirow[t]{2}{*}{ Tracer } & \multirow[t]{2}{*}{ Year } & \multicolumn{2}{|c|}{ No. of patients } & \multirow[t]{2}{*}{ Dose (MBq) } & \multirow[t]{2}{*}{ CT } & \multirow[t]{2}{*}{ Time (min) } & \multirow[t]{2}{*}{ Sens $(\%)$} & \multirow[t]{2}{*}{ PPV (\%) } \\
\hline & & & Total & pHPT + surgery & & & & & \\
\hline Kluijfhout & $18 \mathrm{~F}-\mathrm{FCH}$ & 2015 & 5 & 5 & $2 \mathrm{MBq} / \mathrm{kg}$ & yes & direct & 80 & 100 \\
\hline Michaud & $18 \mathrm{~F}-\mathrm{FCH}$ & 2015 & 17 & 11 & $3 \mathrm{MBq} / \mathrm{kg}$ & yes & direct & 94 & 91 \\
\hline Lezaic & $18 \mathrm{~F}-\mathrm{FCH}$ & 2015 & 43 & 43 & 100 & yes & $5,60,120$ & 95 & 97 \\
\hline Orevi & $11 \mathrm{C}-\mathrm{CH}$ & 2014 & 40 & $?$ & 370 & yes & direct & $?$ & $?$ \\
\hline Lange-Nolde & $18 \mathrm{~F}-\mathrm{DOPA}$ & 2006 & 8 & 8 & $185-300$ & no & 90 & - & - \\
\hline Chicklore & 18F-FDG & 2014 & 43 & 15 & 400 & yes & 90 & 27 & 100 \\
\hline Neumann & $18 \mathrm{~F}-\mathrm{FDG}$ & 1997 & 20 & 20 & $185-370$ & no & 45 & 62 & 62 \\
\hline Neumann & 18F-FDG & 1996 & 21 & 21 & $185-370$ & no & 45 & 86 & 86 \\
\hline Melon & $18 \mathrm{~F}-\mathrm{FDG}$ & 1995 & 7 & 7 & 370 & no & 50 & 22 & 100 \\
\hline Sisson & $18 \mathrm{~F}-\mathrm{FDG}$ & 1994 & 8 & 8 & 370 & no & $?$ & 0 & - \\
\hline Neumann & 18F-FDG & 1994 & 17 & 17 & $185-370$ & no & 45 & 94 & 85 \\
\hline
\end{tabular}

pHPT primary hyperparathyroidism, Sens sensitivity, $P P V$ positive predictive value 
data suggest that $11 \mathrm{C}-\mathrm{CH}$ may be an important tracer in radiographic localization of parathyroid adenomas. The main disadvantage (similar to $11 \mathrm{C}-\mathrm{MET}$ ) is the short half-life of $20 \mathrm{~min}$, which obligates on-site production. This problem has been overcome by the most promising PET tracer thus far, $18 \mathrm{~F}-\mathrm{FCH}$, which has a half-life of $110 \mathrm{~min}$. This tracer is frequently used in the diagnostic work-up and follow-up of patients with prostate cancer and has, therefore, wide availability. So far, three studies have published unique data with excellent preliminary results, even in patients with negative SPECT-CT [27-29]. The advantage of $18 \mathrm{~F}-\mathrm{FCH}$ PET-CT over SPECT-CT is the higher spatial resolution, lower radiation burden (around 6 versus $8 \mathrm{mSv}$ ), and the substantially shorter scanning time with a single acquisition of around $10 \mathrm{~min}$, depending on protocol [44].

Besides PET, several other modalities have been studied for use as a second-line imaging as well. Computed tomography is available in virtually every medical center and has been shown to accurately detect parathyroid adenomas, even in the case of negative conventional imaging $[45,46]$. There is increasing attention to $4 \mathrm{D}-\mathrm{CT}$, which can help clinicians differentiate adenomas from other structures in the neck [47]. Performance has shown to be good, with a sensitivity and PPV of around 80 and $90 \%$, respectively, depending on the investigated population [48, 49]. The major drawback of this technique, which uses multiple phases, is radiation exposure that can be as high as $26 \mathrm{mSv}$ from a single scan [50]. MRI can also be used to localize parathyroid glands. Evidence remains scarce, but one of the largest studies so far showed a sensitivity of $82 \%$ with a PPV of $89 \%$ and a very recent publication also showed promising results [51, 52]. Considering the unique benefits of $18 \mathrm{~F}-\mathrm{FCH}$ PET and MRI, a potentially superior modality would be using the $18 \mathrm{~F}-\mathrm{FCH}$ tracer and a combined PET MRI scanner. Although no studies have been published yet, preliminary results from a pilot-study show excellent results in patients with negative or discordant conventional imaging [Unpublished results Kluijfhout et al.].

Our meta-analysis has several limitations. Like any meta-analysis, its quality is limited by the number and quality of constituent studies. Each individual study included only a small number of patients. As shown by the QUADAS 2 tool, there was also a substantial risk of bias arising from variability in patient selection and reference standard. Moreover, there is concern regarding applicability as various imaging protocols were used. The results of this meta-analysis should therefore be interpreted with caution, and more research, especially in the form of prospective studies, is needed to confirm our observations.

\section{Conclusion}

To our knowledge, this is the first systematic review investigating all PET tracers that have been used for the detection of pathological parathyroid glands. The results of our metaanalysis show that $11 \mathrm{C}$-MET PET has an overall good sensitivity and PPV and may be considered a reliable second-line imaging modality to enable minimally invasive parathyroidectomy. Our literature review suggests that $18 \mathrm{~F}-\mathrm{FCH}$ PET may produce even greater accuracy and should be further investigated using both low-dose CT and MRI for anatomical correlation.

Author contributions All of the authors contributed to the study conception and design and the critical revision of the manuscript.

Wouter P Kluijfhout and Jesse D Pasternak contributed to the acquisition of data.

Wouter P Kluijfhout, Jesse D Pasternak, Frederick T Drake, and Isabel E Allen contributed to the analysis and interpretation of data.

Wouter P Kluijfhout, Jesse D Pasternak, Frederick T Drake, Bart de Keizer, Miguel H Pampaloni, and Insoo Suh contributed to the drafting of the manuscript.

Compliance with ethical standards No funding was received for this work.

This article does not contain any studies with human participants or animals performed by any of the authors.

Conflicts of interest The authors declare that they have no conflict of interest.

Open Access This article is distributed under the terms of the Creative Commons Attribution 4.0 International License (http:// creativecommons.org/licenses/by/4.0/), which permits unrestricted use, distribution, and reproduction in any medium, provided you give appropriate credit to the original author(s) and the source, provide a link to the Creative Commons license, and indicate if changes were made.

\section{References}

1. Fraser WD (2009) Hyperparathyroidism. Lancet 374:145-158

2. Phitayakorn R, McHenry CR (2006) Incidence and location of ectopic abnormal parathyroid glands. Am J Surg 191:418-423

3. Udelsman R, Akerstrom G, Biagini C et al (2014) The surgical management of asymptomatic primary hyperparathyroidism: proceedings of the Fourth International Workshop. J Clin Endocrinol Metab 99:3595-3606

4. Sackett WR, Barraclough B, Reeve TS, Delbridge LW (2002) Worldwide trends in the surgical treatment of primary hyperparathyroidism in the era of minimally invasive parathyroidectomy. Arch Surg 137:1055-1059

5. Irvin GL, Carneiro DM, Solorzano CC (2004) Progress in the operative management of sporadic primary hyperparathyroidism over 34 years. Ann Surg 239:704-708, discussion 708-711

6. Prommegger R, Wimmer G, Profanter C et al (2009) Virtual neck exploration: a new method for localizing abnormal parathyroid glands. Ann Surg 250:761-765

7. Wei WJ, Shen CT, Song HJ et al (2015) Comparison of SPET/CT, SPET and planar imaging using $99 \mathrm{mTc}-\mathrm{MIBI}$ as independent 
techniques to support minimally invasive parathyroidectomy in primary hyperparathyroidism: a meta-analysis. Hell J Nucl Med 18: $127-135$

8. Neumann DR, Esselstyn CB, MacIntyre WJ et al (1993) Parathyroid adenoma localization by PET FDG. J Comput Assist Tomogr 17:976-977

9. Whiting PF, Rutjes AWS, Westwood ME et al (2011) Research and reporting methods accuracy studies. Ann Intern Med 155:529-536

10. DerSimonian R, Laird N (1986) Meta-analysis in clinical trials. Control Clin Trials 7:177-188

11. Higgins JPT, Thompson SG, Deeks JJ, Altman DG (2003) Measuring inconsistency in meta-analyses. BMJ Br Med J 327: $557-560$

12. Sterne JA, Sutton AJ, Ioannidis JP et al (2011) Recommendations for examining and interpreting funnel plot asymmetry in metaanalyses of randomised controlled trials. BMJ 343:d4002

13. Braeuning U, Pfannenberg C, Gallwitz B et al (2015) 11Cmethionine PET/CT after in conclusive 99mTc-MIBI-SPECT/CT for localisation of parathyroid adenomas in primary hyperparathyroidism. NuklearMedizin 54:26-30

14. Hayakawa N, Nakamoto Y, Kurihara K et al (2015) A comparison between 11C-methionine PET/CT and MIBI SPECT/CT for localization of parathyroid adenomas/hyperplasia. Nucl Med Commun 36: 53-59

15. Chicklore S, Schulte KM, Talat N, Hubbard JG, O'Doherty M, Cook GJ (2014) 18F-FDG PET rarely provides additional information to $11 \mathrm{C}$-methionine PET imaging in hyperparathyroidism. Clin Nucl Med 39:237-242

16. Martinez-Rodriguez I, Martinez-Amador N, de Arcocha-Torres M et al (2014) Comparison of 99mTc-sestamibi and 11C-methionine $\mathrm{PET} / \mathrm{CT}$ in the localization of parathyroid adenomas in primary hyperparathyroidism. Rev Esp Med Nucl Imagen Mol 33:93-98

17. Weber T, Maier-Funk C, Ohlhauser D et al (2013) Accurate preoperative localization of parathyroid adenomas with $\mathrm{C}-11$ methionine PET/CT. Ann Surg 257:1124-1128

18. Chun IK, Cheon GJ, Paeng JC, Kang KW, Chung JK, Lee DS (2013) Detection and characterization of parathyroid adenoma/ hyperplasia for preoperative localization: comparison between 11C-Methionine PET/CT and 99mTc-sestamibi scintigraphy. Nucl Med Mol Imaging 47:166-172

19. Schalin-Jantti C, Ryhanen E, Heiskanen I et al (2013) Planar scintigraphy with 123I/99mTc-sestamibi, 99mTc-sestamibi SPECT/CT, 11C-methionine PET/CT, or selective venous sampling before reoperation of primary hyperparathyroidism? J Nucl Med 54:739-747

20. Oksuz MO, Dittmann H, Wicke C et al (2011) Accuracy of parathyroid imaging: a comparison of planar scintigraphy, SPECT, SPECT-CT, and C-11 methionine PET for the detection of parathyroid adenomas and glandular hyperplasia. Diagn Interv Radiol 17: 297-307

21. Weber T, Cammerer G, Schick C et al (2010) C-11 methionine positron emission tomography/computed tomography localizes parathyroid adenomas in primary hyperparathyroidism. Horm Metab Res 42:209-214

22. Herrmann K, Takei T, Kanegae K et al (2009) Clinical value and limitations of $[11 \mathrm{C}]-$ methionine PET for detection and localization of suspected parathyroid adenomas. Mol Imaging Biol 11:356-363

23. Tang BNT, Moreno-Reyes R, Blocklet D et al (2008) Accurate preoperative localization of pathological parathyroid glands using 11C-methionine PET/CT. Contrast Media Mol Imaging 3:157-163

24. Beggs AD, Hain SF (2005) Localization of parathyroid adenomas using 11C-methionine positron emission tomography. Nucl Med Commun 26:133-136

25. Otto D, Boerner AR, Hofmann M et al (2004) Pre-operative localisation of hyperfunctional parathyroid tissue with 11Cmethionine PET. Eur J Nucl Med Mol Imaging 31:1405-1412
26. Sundin A, Johansson C, Hellman P et al (1996) Pet and parathyroid L-[carbon-11]methionine accumulation in hyperparathyroidism. J Nucl Med 37:1766-1770

27. Kluijfhout WP, Vorselaars WMCM, Vriens MR et al (2015) Enabling minimal invasive parathyroidectomy for patients with primary hyperparathyroidism using Tc-99m-sestamibi SPECTCT, ultrasound and first results of 18F-fluorocholine PET-CT. Eur J Radiol 84:1745-1751

28. Michaud L, Balogova S, Burgess A et al (2015) A pilot comparison of $18 \mathrm{~F}$-fluorocholine PET/CT, ultrasonography and $123 \mathrm{I} / 99 \mathrm{mTc}-$ sestaMIBI dual-phase dual-isotope scintigraphy in the preoperative localization of hyperfunctioning parathyroid glands in primary or secondary hyperparathyroidism: influence of thyroid anomalies. Medicine (Baltimore) 94, e1701

29. Rep S, Lezaic L, Kocjan T et al (2015) Optimal scan time for evaluation of parathyroid adenoma with [18F]-fluorocholine PET/ CT. Radiol Oncol 49:327-333

30. Orevi M, Freedman N, Mishani E, Bocher M, Jacobson O, Krausz Y (2014) Localization of parathyroid adenoma by 11C-Choline PET/CT: preliminary results. Clin Nucl Med 39:1033-1038

31. Lange-Nolde A, Zajic T, Slawik M et al (2006) PET with 18F-DOPA in the imaging of parathyroid adenoma in patients with primary hyperparathyroidism: a pilot study. NuklearMedizin 45:193-196

32. Neumann DR, Esselstyn CBJ, MacIntyre WJ et al (1997) Regional body FDG-PET in postoperative recurrent hyperparathyroidism. J Comput Assist Tomogr 21:25-28

33. Neumann DR, Esselsteyn CB, MacIntyre WJ et al (1996) Comparison of FDG-PET and sestamibi-SPECT in primary hyperparathyroidism. J Nucl Med 37:1809-1815

34. Melon P, Luxen A, Hamoir E et al (1995) Fluorine-18fluorobeoxyglucose positron emission tomography for preoperative parathyroid imaging in primary hyperparathyroidism. Eur $\mathrm{J}$ Nucl Med 22:556-558

35. Sisson JC, Thompson NW, Ackerman RJ et al (1994) Use of 2-[F18]-fluoro-2-deoxy-D-glucose PET to locate parathyroid adenomas in primary hyperparathyroidism. Radiology 192:280

36. Neumann DR, Esselstyn CBJ, MacIntyre WJ et al (1994) Primary hyperparathyroidism: preoperative parathyroid imaging with regional body FDG PET. Radiology 192:509-512

37. Michaud L, Burgess A, Huchet V et al (2014) Is (18)f-fluorocholinepositron emission tomography/computerized tomography a new imaging tool for detecting hyperfunctioning parathyroid glands in primary or secondary hyperparathyroidism? J Clin Endocrinol Metab 99:4531-4536

38. Lezaic L, Rep S, Sever MJ et al (2014) 18F-Fluorocholine PET/CT for localization of hyperfunctioning parathyroid tissue in primary hyperparathyroidism: a pilot study. Eur J Nucl Med Mol Imaging 41:2083-2089

39. Caldarella C, Treglia G, Isgro MA et al (2013) Diagnostic performance of positron emission tomography using $11 \mathrm{C}$-methionine in patients with suspected parathyroid adenoma: a meta-analysis. Endocrine 43:78-83

40. Cheung K, Wang TS, Farrokhyar F et al (2012) A meta-analysis of preoperative localization techniques for patients with primary hyperparathyroidism. Ann Surg Oncol 19:577-583

41. Treglia G, Sadeghi R, Schalin-Jantti C et al (2015) Detection rate of $99 \mathrm{~m}$ Tc-MIBI single photon emission computed tomography (SPECT)/CT in preoperative planning for patients with primary hyperparathyrodism: a meta-analysis. Head Neck. doi:10.1002/ hed.24027

42. Hoang JK, Sung W, Bahl M et al (2014) How to perform parathyroid 4D CT: tips and traps for technique and interpretation. Radiology 270:15-24

43. Mahajan A, Starker LF, Ghita M et al (2012) Parathyroid fourdimensional computed tomography: evaluation of radiation dose 
exposure during preoperative localization of parathyroid tumors in primary hyperparathyroidism. World J Surg 36:1335-1339

44. Kluijfhout WP, Vriens MR, Valk GD et al (2015) $18 \mathrm{~F}$ Fluorocholine PET-CT enables minimal invasive parathyroidectomy in patients with negative sestamibi SPECT-CT and ultrasound: a case report. Int J Surg Case Rep 13:73-75

45. Lubitz CC, Stephen AE, Hodin RA, Pandharipande P (2012) Preoperative localization strategies for primary hyperparathyroidism: an economic analysis. Ann Surg Oncol 19:4202-4209

46. Zald PB, Hamilton BE, Larsen ML, Cohen JI (2008) The role of computed tomography for localization of parathyroid adenomas. Laryngoscope 118:1405-1410

47. Rodgers SE, Hunter GJ, Hamberg LM et al (2006) Improved preoperative planning for directed parathyroidectomy with 4dimensional computed tomography. Surgery 140:932-941

48. Hunter GJ, Schellingerhout D, Vu TH et al (2012) Accuracy of four-dimensional CT for the localization of abnormal parathyroid glands in patients with primary hyperparathyroidism. Radiology 264:789-795

49. Kukar M, Platz TA, Schaffner TJ et al (2015) The use of modified four-dimensional computed tomography in patients with primary hyperparathyroidism: an argument for the abandonment of routine sestamibi single-positron emission computed tomography (SPECT). Ann Surg Oncol 22:139-145

50. Starker LF, Mahajan A, Bjorklund P, Sze G, Udelsman R, Carling T (2011) 4D parathyroid CT as the initial localization study for patients with de novo primary hyperparathyroidism. Ann Surg Oncol 18:1723-1728

51. Sacconi B, Argirò R, Diacinti D et al (2015) MR appearance of parathyroid adenomas at $3 \mathrm{~T}$ in patients with primary hyperparathyroidism: what radiologists need to know for pre-operative localization. Eur Radiol. doi:10.1007/s00330-015-3854-5

52. Gotway MB, Reddy GP, Webb WR et al (2001) Comparison between MR imaging and 99mTc MIBI scintigraphy in the evaluation of recurrent of persistent hyperparathyroidism. Radiology 218:783-790 